in vivo $32: 221-229(2018)$

doi:10.21873/invivo.11228

Review

\title{
Non Lipomatous Benign Lesions Mimicking Soft-tissue Sarcomas: A Pictorial Essay
}

\author{
ALESSANDRO CORAN ${ }^{1}$, GIOVANNA ORSATTI ${ }^{2}$, FILIPPO CRIMİ ${ }^{2}$, MARCO RASTRELLI $^{3}$, \\ ANTONIO DI MAGGIO ${ }^{1}$, ALBERTO PONZONI $^{4}$, SHADY ATTAR $^{2}$ and ROBERTO STRAMARE ${ }^{2}$ \\ ${ }^{1}$ Department of Radiology and Medical Physics, Oncologic Radiology Unit, \\ Veneto Institute of Oncology, IOV-IRCCS, Padua, Italy; \\ ${ }^{2}$ Radiology Unit, Department of Medicine-DIMED, University Hospital of Padova, Padua, Italy; \\ ${ }^{3}$ Department of Surgical Oncology, Surgical Oncology Unit, Veneto Institute of Oncology, IOV-IRCCS, Padua, Italy; \\ ${ }^{4}$ Department of Radiology, Azienda Ospedaliera di Padova, Padua, Italy
}

\begin{abstract}
The incidental finding of soft tissue masses is a challenge for the radiologist. Benign and malignant lesions can be differentiated relying on patient history, symptoms and mostly with the help of imaging. Ultrasound (US), computed tomography $(C T)$ and magnetic resonance imaging (MRI) become fundamental in order to distinguish these lesions but the radiologist needs to know the main characteristics of benign soft tissue masses and sarcomas. Herein, we present a pictorial review of lesions mimicking soft tissue sarcomas features.
\end{abstract}

Pseudosarcomas are benign soft tissue mimic lesions representing a challenge for radiologists since they can erroneously be mistaken for malignancies. Every misdiagnosis may bring to an over-treatment or vice versa to an under-treatment, therefore, the knowledge of the features of the most common soft tissue mimic lesions is crucial to address patients to the correct therapeutic path.

Mimic lesions may have a neoplastic or non-neoplastic origin and we categorised them on the basis of the World Health Organisation (WHO) 2013 sarcomas classification (Table I) (1).

Every lesion may have predominant ultrasound (US), computed tomography (CT) or magnetic resonance imaging

This article is freely accessible online.

Correspondence to: Marco Rastrelli, Department of Surgical Oncology, Surgical Oncology Unit, Veneto Institute of Oncology, IOV-IRCCS, Padua, Italy. Tel: +39 0498212137, e-mail: marco.rastrelli@iov.veneto.it, shadypt86@gmail.com

Key Words: Sarcoma, mimics, MRI, review.
(MRI) features that should be recognized in order to avoid a misdiagnosis. Patient history and symptoms are also crucial to distinguish these benign lesions from soft tissue sarcomas.

\section{Neoplastic Soft Tissue-Mimicing Lesions}

Ganglion and synovial cysts. These mucinous liquidcontaining lesions are thought to result from chronic joint effusion and herniation of the intra-articular fluid delimited by synovial membrane (synovial cyst) or from micro-injuries that cause synovial-capsular interface reaction creating a liquid formation with pseudo-synovial membrane (ganglion cyst). The specific locations of these lesions, i.e. popliteal region for synovial cyst and wrist region for ganglion cysts, may suggest the correct diagnosis.

However, the presentation in atypical sites and an unusual radiological aspect can arise the suspect of malignancies. Synovial cysts appear as well-defined uniloculated or multiloculated lesions with anechoic content on US examination and liquid densitometry on CT scan. On MRI, they show bright signal intensity on $\mathrm{T} 2$ weighted images and low signal intensity on $\mathrm{T} 1$ weighted images with exclusive enhancement of the capsule after gadolinium injection (Figure 1) (2).

Myositis ossificans. Myositis ossificans can be defined as a benign heterotopic ossification typically occurring in large muscles of extremities in young adults, usually associated with a local trauma. It develops a "zonal" organization: a central fibroblastic proliferation focus, an intermediate area with immature osteoid matrix and a peripheral zone with mature lamellar bone. Calcifications can be seen on plain film and CT scans within 2-6 weeks; US shows a hypoechoic mass with 
Table I. Neoplastic and non-neoplastic lesions that mimic malignancies and main differential diagnoses.

Neoplastic

Ganglion and synovial cyst

Myositis ossificans

Synovial chondromatosis

hemangioma

Elastofibroma

Nodular Fasciitis

Intramuscular Myxoma

Schwannoma

Non neoplastic

Infection

Hematoma

Arteriovenous malformation

Morton's Neuroma

\author{
Giant cells tumor, synovial sarcoma, myxoid sarcomas \\ Synovial sarcoma, chondrosarcoma, paraosteal osteosarcoma \\ Chondrosarcoma, synovial sarcoma. \\ Angiosarcoma, epithelioid haemangioendothelioma, Kaposi sarcoma \\ Desmoid fibromatosis, fibrosarcoma, undifferentiated pleomorphic sarcoma \\ Desmoid fibromatosis, fibrosarcoma, undifferentiated pleomorphic sarcoma \\ Fibromyxoid sarcoma, myxoid liposarcoma, undifferentiated pleomorphic sarcoma \\ Malignant peripheral nerve sheath tumors, undifferentiated pleomorphic sarcoma
}

Leiomyosarcoma, necrotic malignant lesions

Haemorrhagic malignant tumors, leiomyosarcoma

Angiosarcoma, epithelioid haemangioendothelioma, Kaposi sarcoma

Desmoid fibromatosis, fibrosarcoma lamellar hyperreflectivity at the periphery. On MRI, early lesions are isointense to muscle in T1 weighted images and heterogeneously hyperintense with surrounding soft tissue edema in T2 weighted images; diffuse contrast enhancement is usually seen. As the lesion develops, a peripheral low signal rim due to bone deposition appears both in T1 weighted images and T2 weighted images (Figure 2) (2, 3).

Synovial chondromatosis. Primary synovial chondromatosis is a proliferative and metaplasic disorder of the synovium of joints, tendon sheaths or bursae with resultant formation of multiple intra-articular chondral nodules. It most frequently affects the knee in men in the $4^{\text {th }}$ or $5^{\text {th }}$ decade, followed by the hip. Secondary synovial chondromatosis is associated with joint abnormalities as result of trauma or arthritic conditions.

$\mathrm{X}$-ray and CT show multiple intra-articular calcifications in $70-95 \%$ of cases; CT is more sensitive in detecting both calcifications and bone erosions.

On MRI, cartilaginous nodules appear iso-hypointense to muscle on T1 weighted images and highly hyperintense on T2 weighted images, with variable focal areas of low signal in all sequences depending on the degree of mineralization; MRI also can reveal the surrounding soft-tissue involvement (Figure 3) (4).

Hemangioma. Classified among vascular abnormalities, these benign neoplastic lesions are typical of infancy and childhood. Characterized by non-atypical endothelial cells hyperplasia and increased number of vessels with a variable fatty component interposed, their natural history consists of an early proliferative phase followed by a later regression. Head and neck are the typical locations. In US they present as highly vascularized hypo-anechogenic masses. CT shows multilobulated lesions iso-attenuating to muscles while on MRI they appear intermediately intense on T1-weighted images and hyper-intense on T2-weighted images because of both fat and liquid content; fat-saturated T2-weighted sequences can distinguish between the two components. After contrast injection hemangiomas present a variable early enhancement depending on the arterial high-flow component and usually a homogeneous enhancement in late phases due to the venous component (Figure 4) (5). Fluid-fluid levels due to the venous low flow component and internal septa can be present.

Elastofibroma. Elastofibromas are benign, slow-growing tumors usually occurring in women in the sixth decade or older (1).

Composed of elastic fibres, collagen bundles, fibroblasts and adipocytes, elastofibromas are typically found at the infero-medial border of the scapula between latissimus dorsi, serratus anterior and chest wall. Their genesis is probably related to friction between scapula and chest wall.

On CT elastofibromas have similar attenuation to muscles with contextual low-attenuating fatty streaks. MRI shows uncapsulated masses with alternating bands of different signal intensity. Collagen and elastic fibres appear hypointense in all sequences while adipose components are hyperintense both on $\mathrm{T} 1$ weighted images and on $\mathrm{T} 2$ weighted images and hypointense on fat-saturated sequences. Heterogeneous enhancement is usually seen after gadolinium administration (Figure 5) (6, 7).

Nodular fasciitis. Nodular fasciites consist of small, benign, rapidly-growing fibrous lesions usually affecting young adults (20-40 years). Upper extremities, especially the volar aspect of the forearm, are the most frequent locations $(46 \%$ of cases) (1); other less common sites are trunk, neck and lower extremities. 


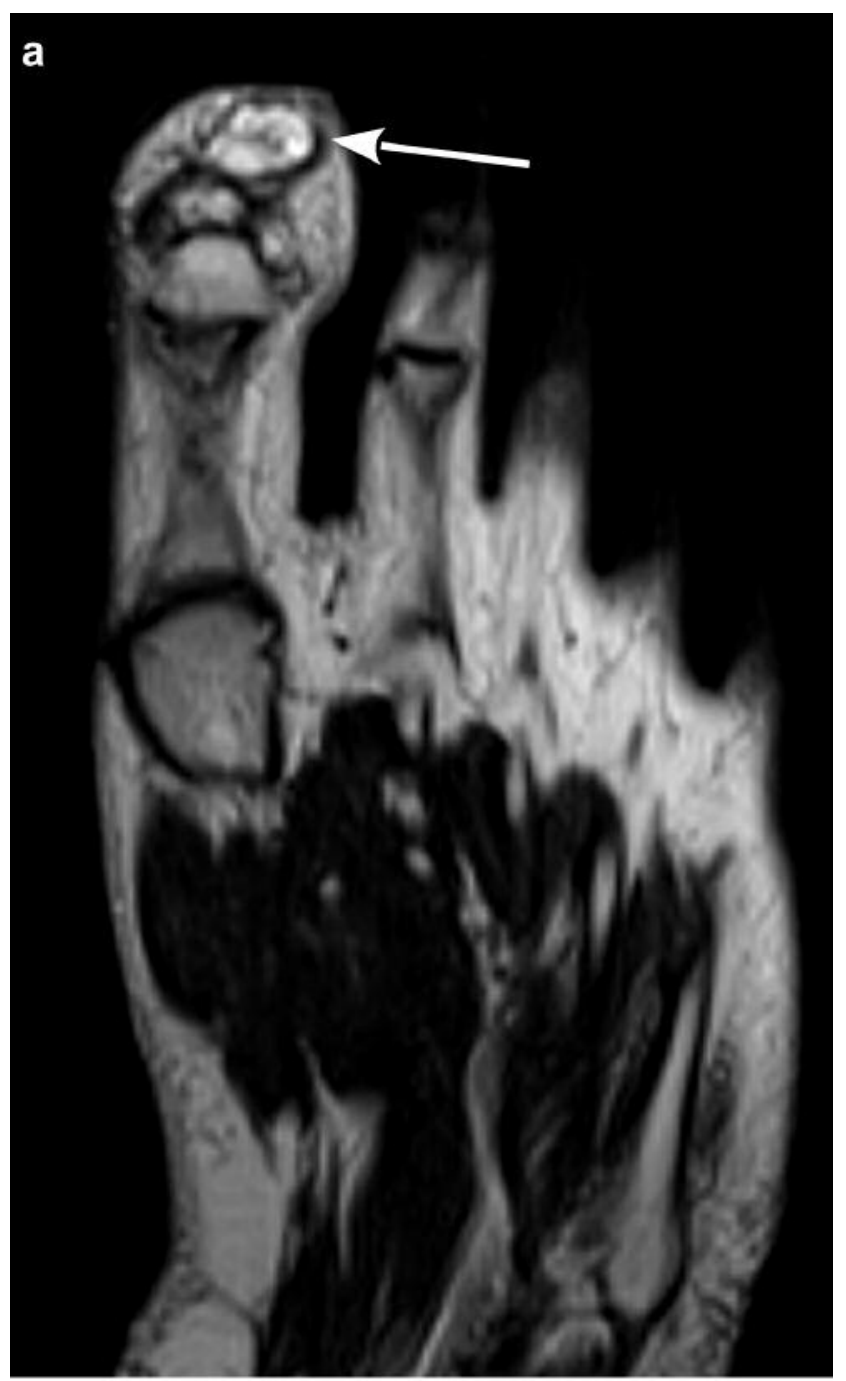

b

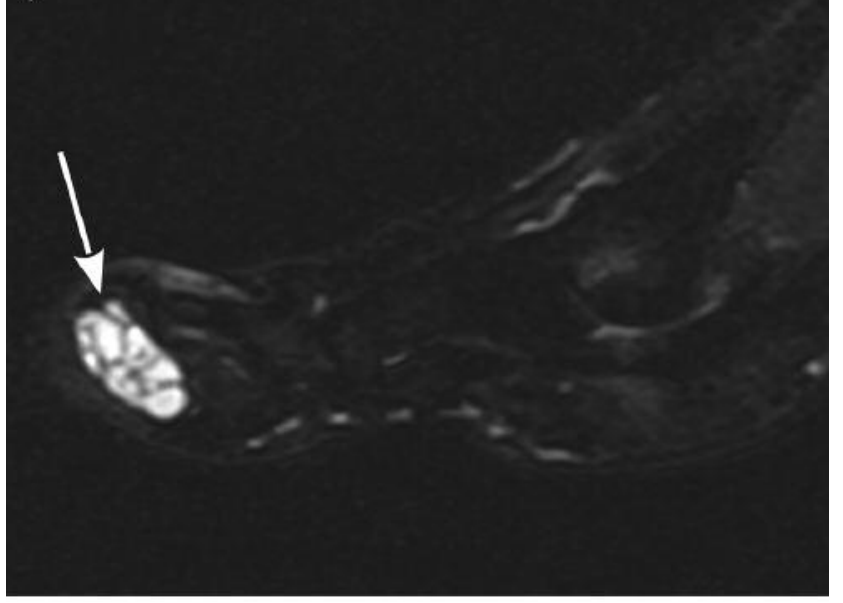

Figure 1. Synovial tendon cyst: a) Axial T2 weighted MRI scan showing a hyper-intense multi-lobulated mass of the first toe (white arrow); $b$ ) Sagittal STIR MRI scan highlighting the fluid content of the same lesion (white arrow). Synovial tendon cyst was found at histopathology.
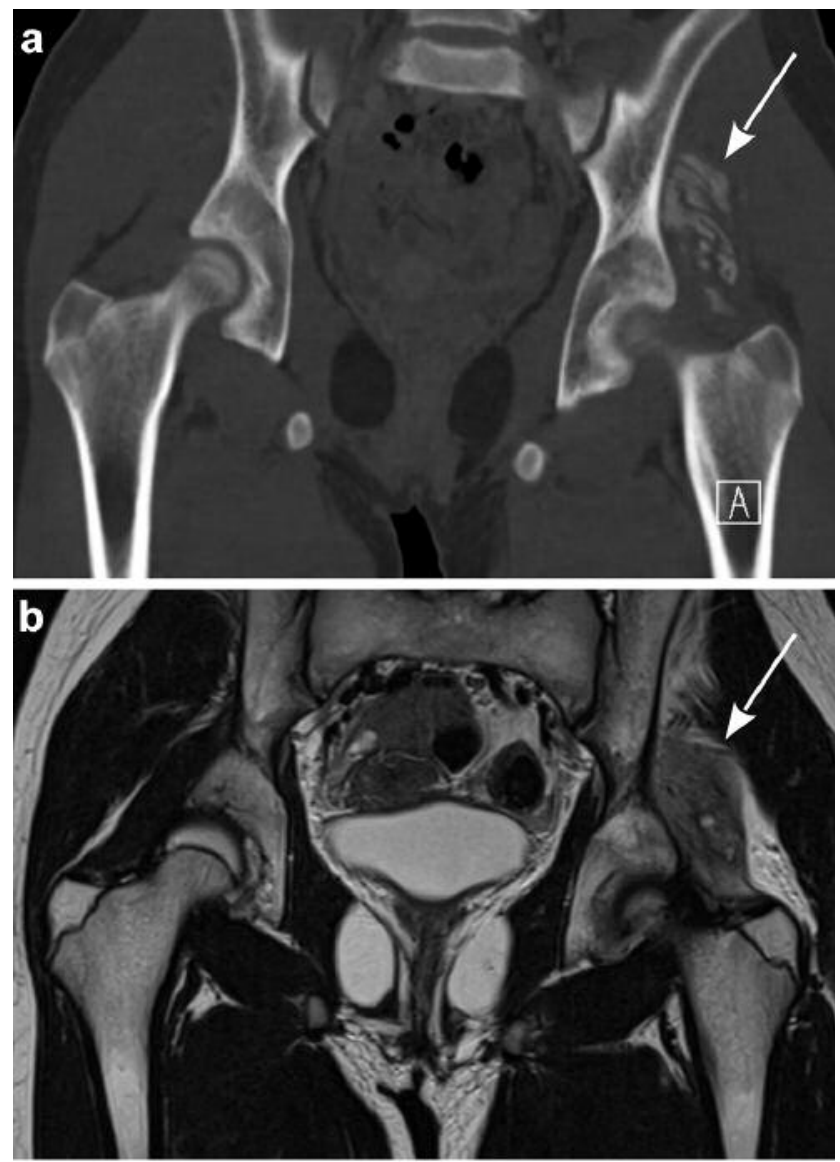

Figure 2. Myositis ossificans: a) Coronal CT scan showing an inhomogeneous calcific mass (white arrow) of the left gluteus minimus; b) Coronal T2 weighted MRI scan shows an enlargement of the gluteus minimus with an inhomogeneous hyper-intense signal (white arrow) and focal areas of signal void. Myositis ossificans was proven with biopsy.

These fibrous nodules consist of proliferations of fibroblasts and myofibroblasts included in variable myxoid or fibrous stroma. According to the main component of the lesion, three pathological subtypes are defined: cellular, myxoid or fibrous. US examination reveals well-defined, solid nodes with variable echogenicity. MRI shows masses extending along fascia, whose appearance reflects the different subtypes; cellular lesions are iso-intense in T1 weighted images and hyper-intense in T2 weighted images while fibrous lesions due to their collagenous components are invariably hypo-intense. Diffuse contrast enhancement is usually seen (Figure 6) (6).

Intramuscular myxoma. Myxomas are composed of myxoid tissue containing collagen fibres and undifferentiated stellate cells; in musculoskeletal system they often have 

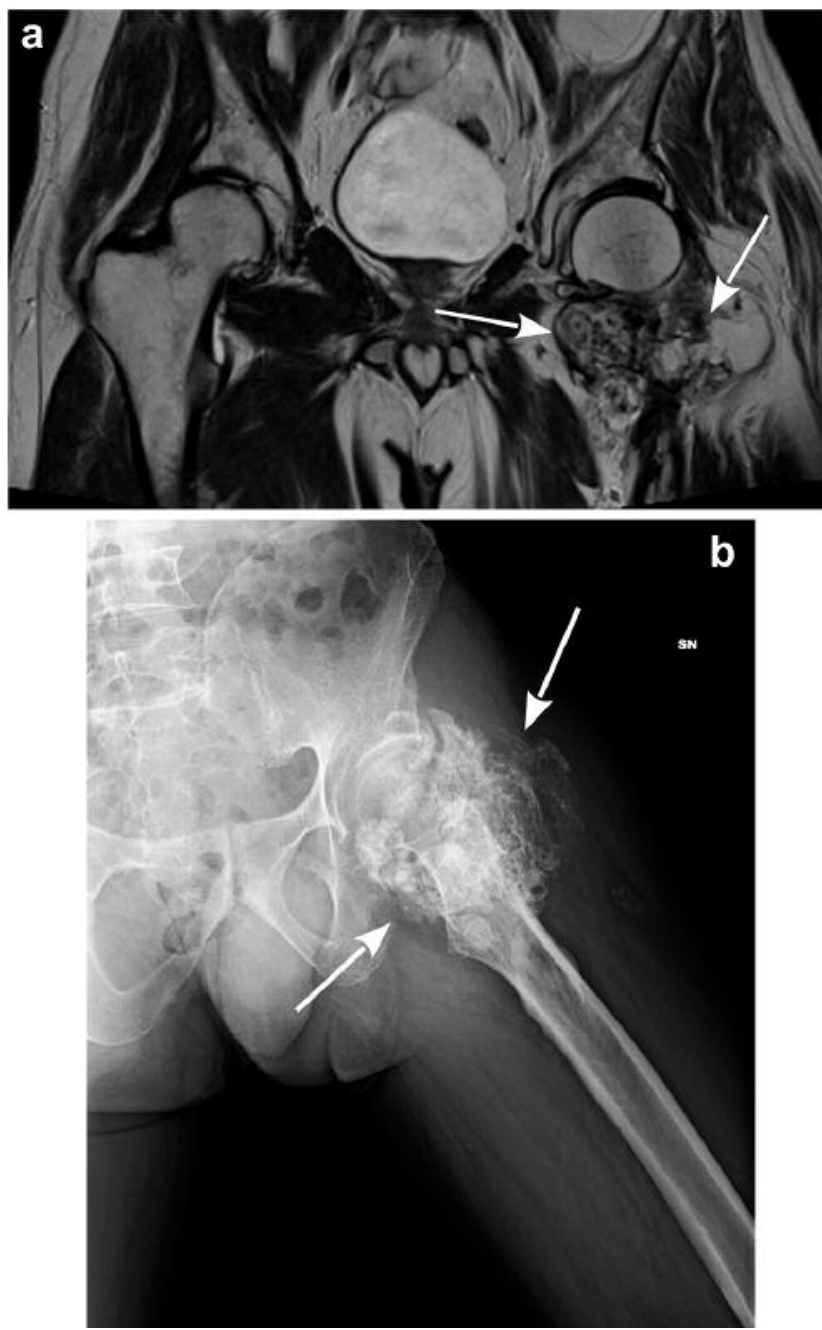

Figure 3. Synovial chondromatosis: a) Coronal T2 weighted MRI scan showing an inhomogeneous hyper-intense lesion (white arrows) with hypo-intense spots inside compatible with calcifications; $b) X$-ray showing diffusely calcific mass (white arrows). Synovial chondromatosis was found at histopathology.

intramuscular location and can be multiple. The clinical presentation as enlarging mass, usually in 40-70 year-old women or men, may arise the suspect of malignancy. US appearance consists of well-defined inhomogeneous hypoanechoic mass with hyperchogenic fatty rim and occasionally with the presence of the "fatty cap" (triangolariform adipose tissue at the myxoma poles). CT shows soft tissue masses with uniform attenuation in unenhanced scan; contrast enhancement is limited to septa and wall in half cases and diffuse in the whole lesion in the other half. Myxomas in MRI demonstrate a low signal on T1 weighted images and a bright and mostly homogeneous signal on T2 weighted images; the lesion is usually surrounded by an hyperintense
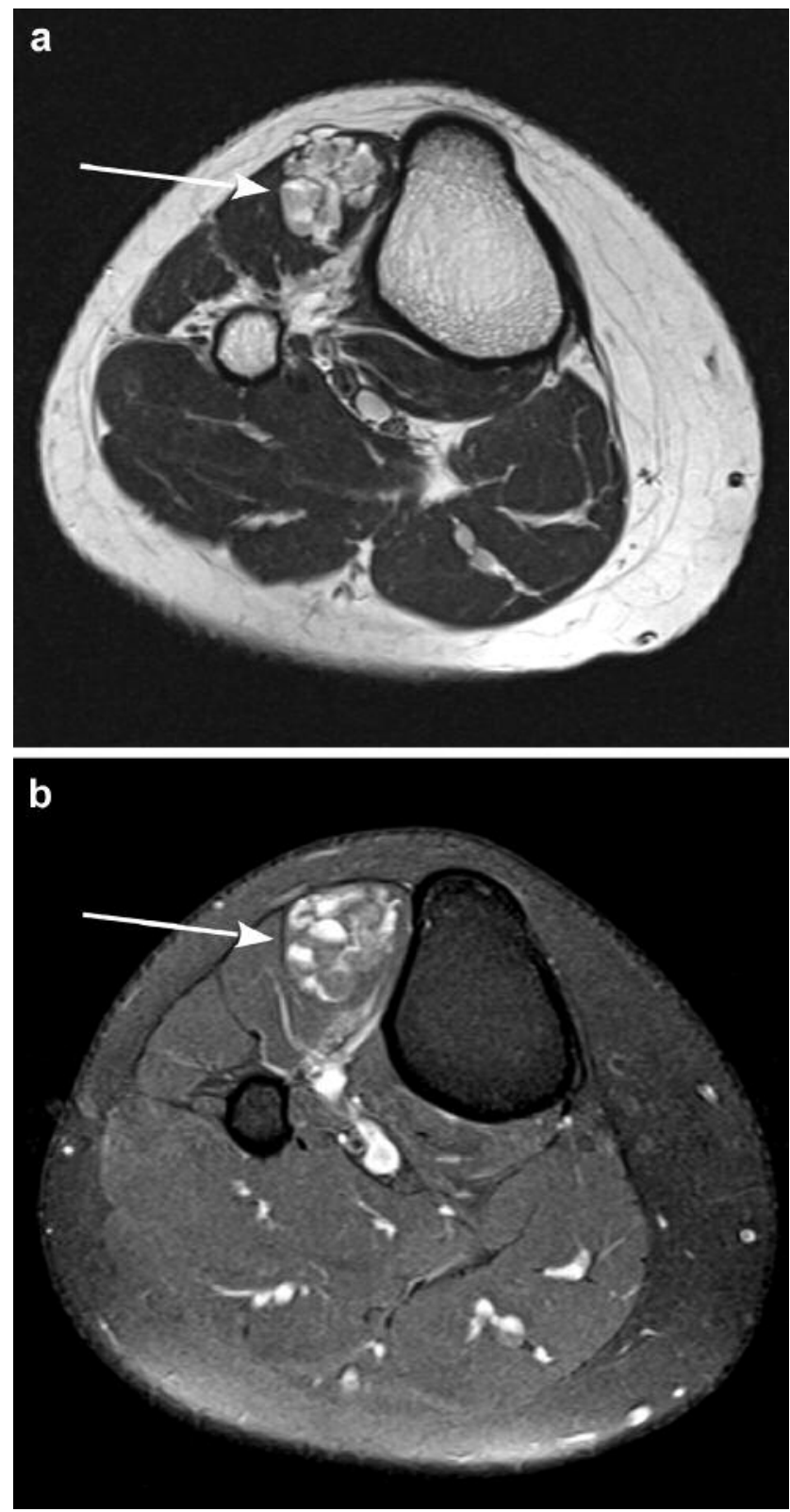

Figure 4. Hemangioma: a) Axial T2 weighted MRI scan showing a hyperintense multi-lobulated mass of the tibialis anterior (with arrow) with multiple fluid-fluid levels; $b$ ) The same lesion (white arrow) on axial fatsaturated post-gadolinium T1 weighted MRI scan showing inhomogeneous contrast enhancement in the venous-phase and fluid-fluid levels. Later contrastographic phases demonstrated a progressive filling of the lesion (not shown). Hemangioma was found at histopathology.

fat ring in non-fat saturated sequences and may have a "fatty cap", as described in the ultrasound features. Peri-lesional leakage of myxomatous tissue can be seen as bright signal in STIR sequences (Figure 7). The contrast enhancement pattern with MRI is the same described for CT (8). 

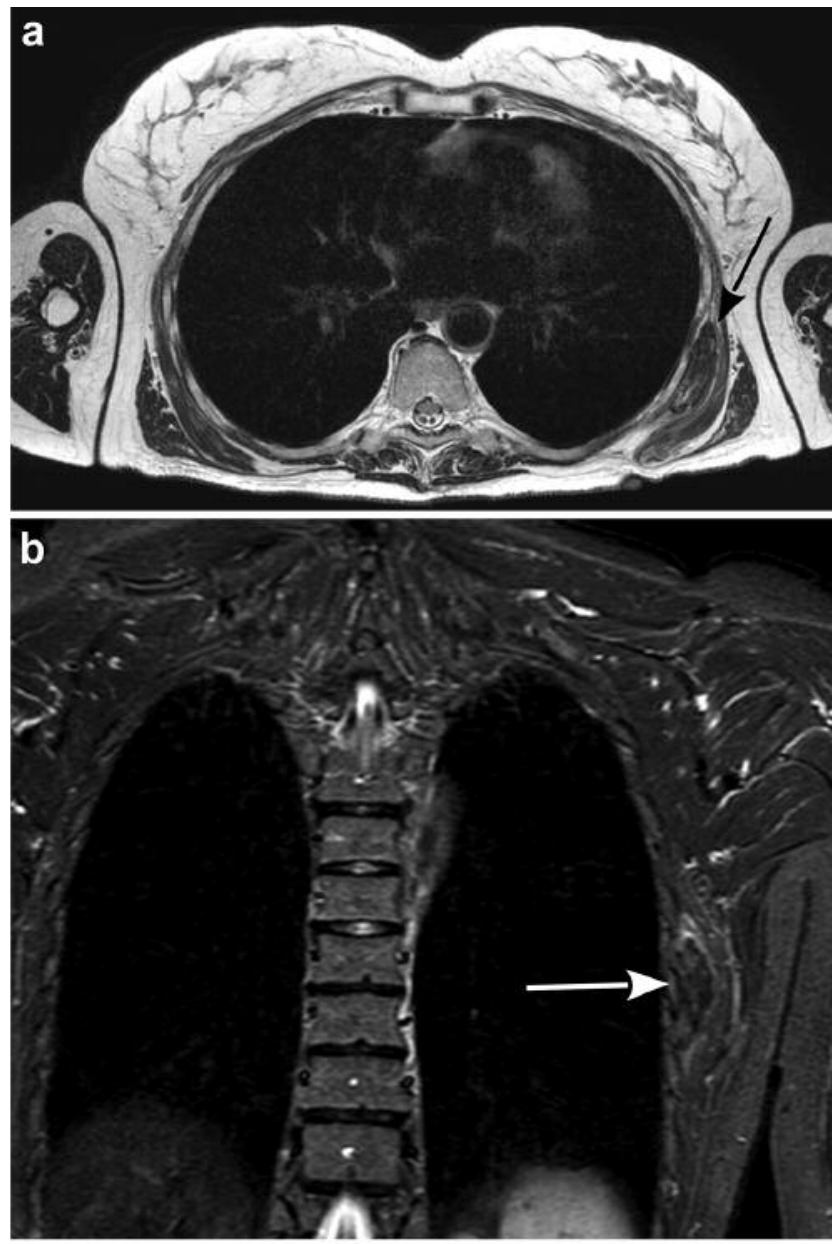

Figure 5. Elastofibroma: a) Axial T2 weighted MRI scan showing hypointense subscapular formation (black arrow) between left serratus anterior muscle and chest wall, with hyper-intense lines consistent with fat tissue; $b$ ) Coronal STIR MRI image confirming the fat component of the lesion (white arrow). Elastofibroma was found at histopathology.

Schwannoma. Schwannomas are benign peripheral nerve sheath tumors growing along the nerve. They are usually eccentric, encapsulated by epineurium and composed of spindle cells and myxoid tissue sometimes with a fibrocollagenous core. Schwannomas affect mostly 20-30 year-old men and women, equally. On US examination they appear as well delimited fusiform hypoechoic masses with the long axis following the nerve course (string sign). CT scan shows inhomogeneous hypo-attenuating masses with heterogeneous diffuse or peripheral enhancement after intravenous iodine contrast injection. In T1 weighted images schwannomas appear iso/hypointense to muscles while in T2 weighted images a bright non-homogeneous signal can be detected, sometimes with a central low intensity fibrous core (target sign) or with a hyperintense fatty rim (split fat sign).
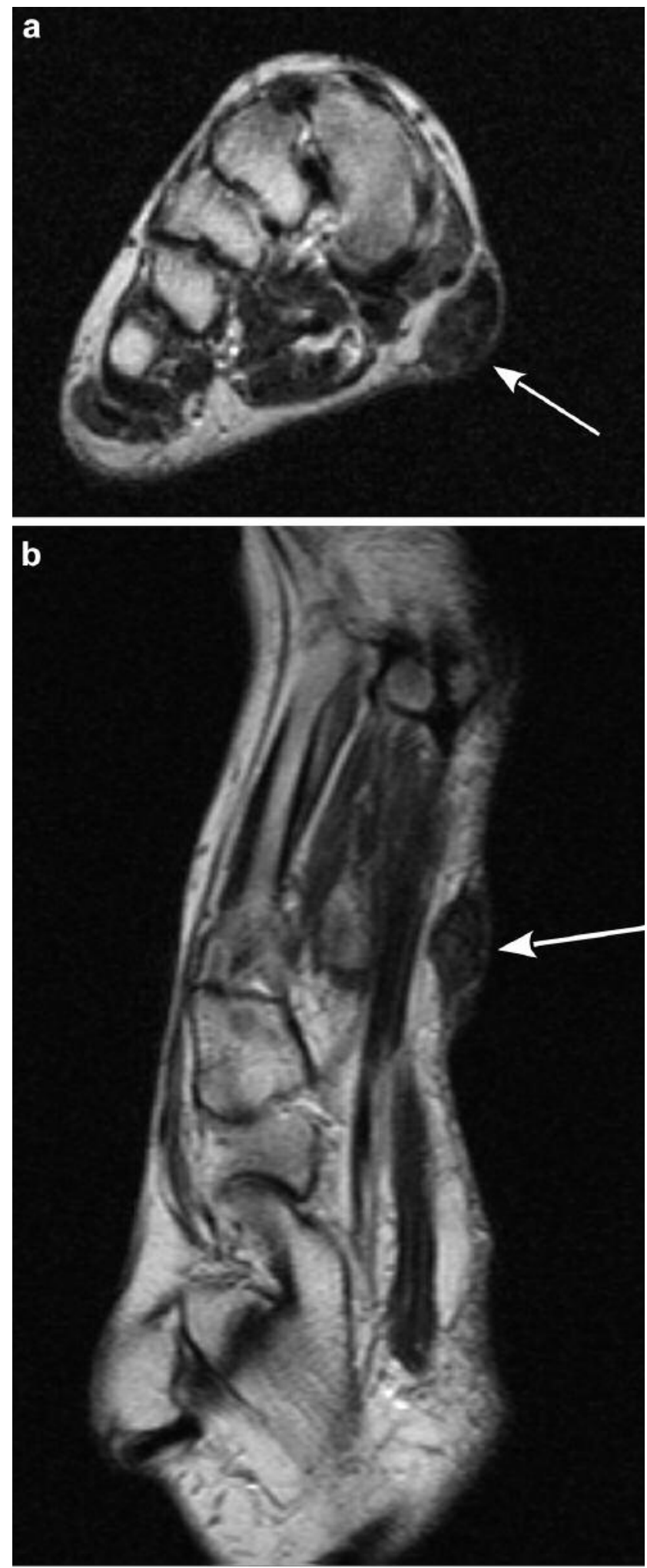

Figure 6. Nodular fasciitis: a) Coronal and b) sagittal T2 weighted MRI scan showing inhomogeneous hypo-intense nodule (white arrows) on the medial side of the plantar fascia, extended to subcutaneous tissue. Nodular fasciitis was found at histopathology. 

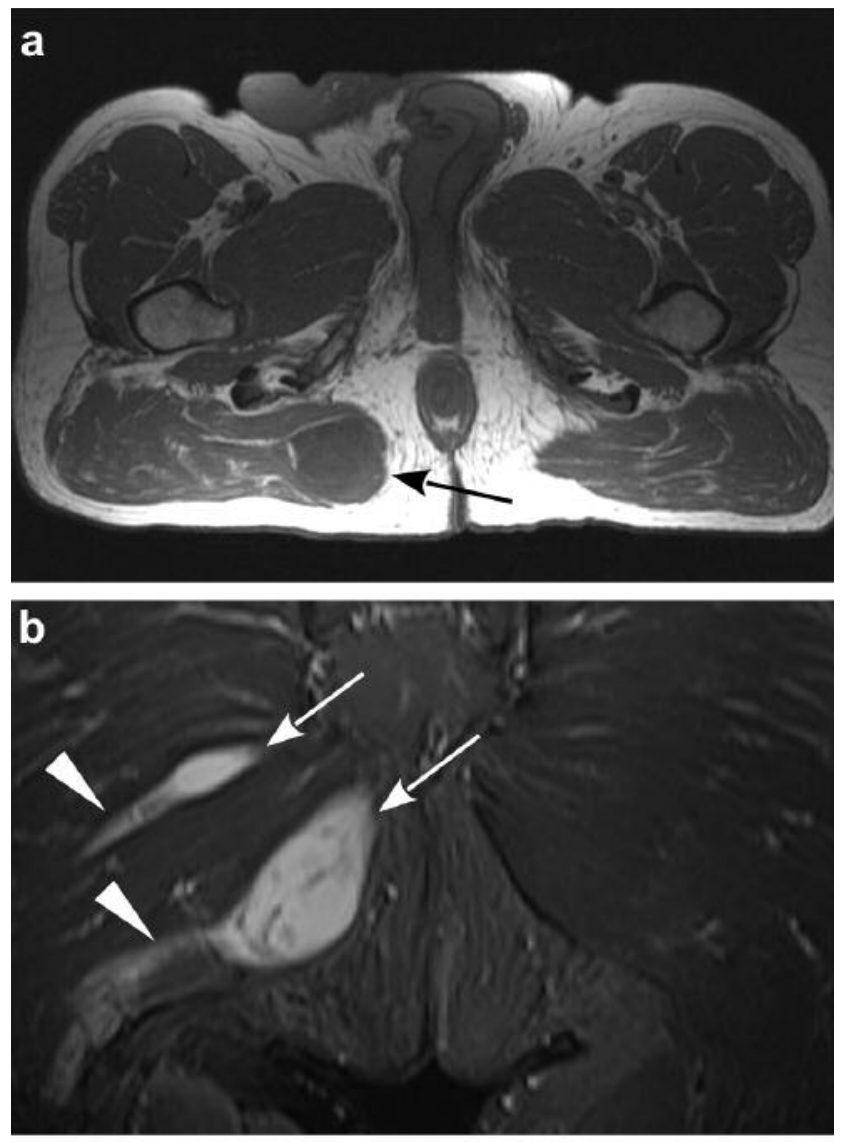

Figure 7. Intramuscular myxoma: a) Axial T1 weighted MRI scan showing a hypo-intense nodule (black arrow) in the gluteus maximus muscle surrounded by a hyper-intense ring (fatty cap); b) Coronal STIR MRI scan showing the previously described lesion and another smaller nodule cranially (white arrows) both with homogeneous hyper-intense signal and peri-lesional hyper-intense offshoots (white arrowheads), consistent with leakage of myxomatous tissue. Intramuscular myxomas were found at histopathology.

Gadolinium enhancement in MRI resembles what has been described above for CT (Figure 8) $(7,8)$.

\section{Non-neoplastic Soft Tissue Mimic Lesion}

Infection. Infections of joints, bones and soft tissues may provide a diagnostic challenge when presenting as a soft tissue mass, especially if accompanied by slight symptoms. Bacterial and mycobacterial abscesses usually present a necrotic core, an irregular and thick highly vascularized wall containing inflammatory cells and edema of surrounding tissues. US examination shows a hypo-anechoic core with irregular vascularized border on Color-Doppler module while, on CT scans, the central hypodense core is circumscribed by a contrast-enhanced rim. The necrotic core
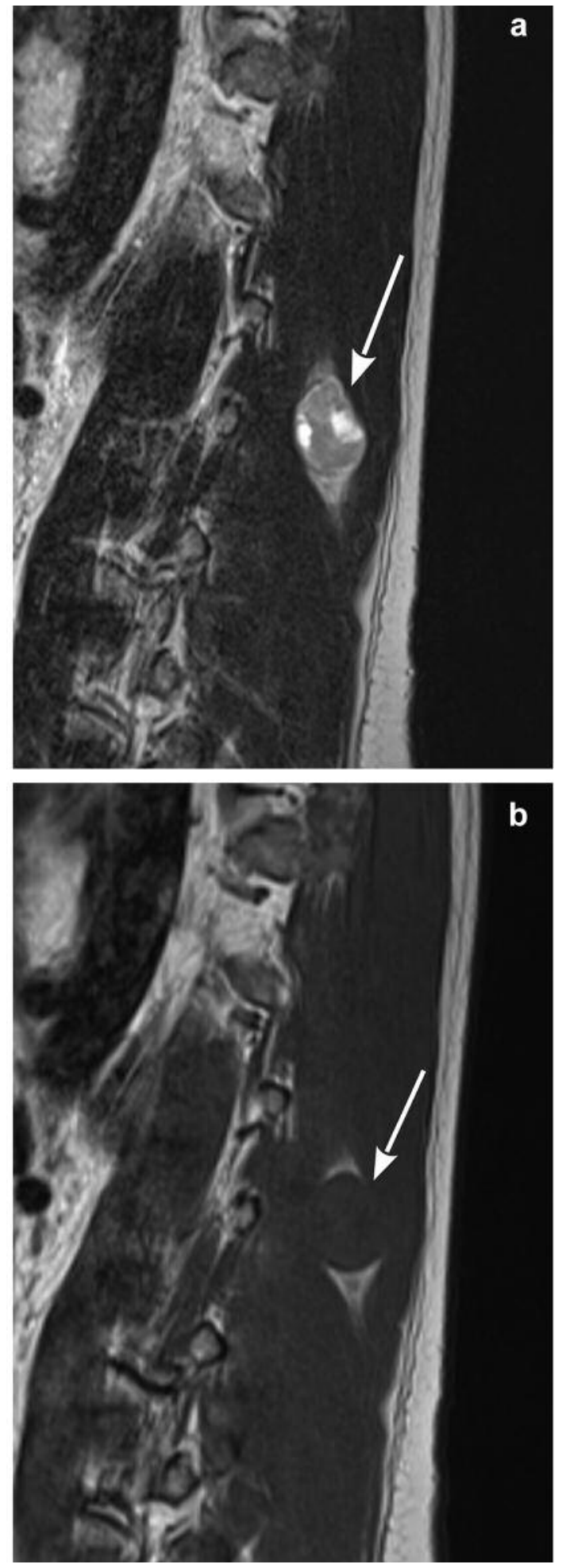

Figure 8. Intramuscular schwannoma: a) Sagittal T2 weighted MRI scan showing an inhomogeneous lesion (white arrow) in the paravertebral muscles with a low intensity fibrous core (target sign); b) Sagittal T1 weighted MRI scan of the same area showing the hyper-intense fatty rim of the lesion (split fat sign). Intramuscular schwannoma was found at histopathology. 

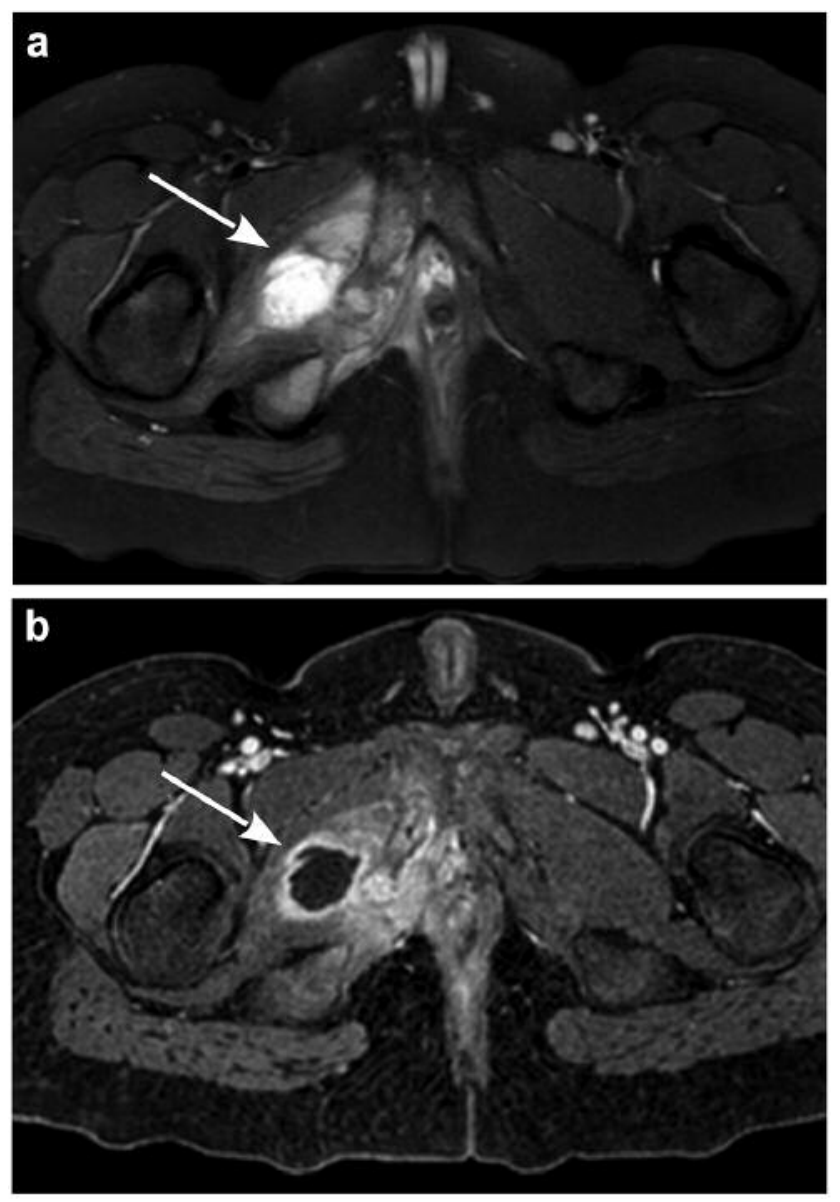

Figure 9. Muscular abscess: a) Axial STIR MRI scan showing a hyperintense lesion of the right external obturator muscle (white arrow) with surrounding edema; $b$ ) Axial T1 weighted MRI scan with fat saturation and after gadolinium injection showing the same area with a rim of peripheral contrast enhancement in venous phase (white arrow). Muscular abscess was diagnosed with needle biopsy.

on MRI is hyperintense in T2 weighted images while the T1 weighted signal depends on the proteic content of the lesion. After gadolinium intravenous injection a vivid enhancement of the wall is seen (Figure 9) (2).

Hematoma. Soft tissue hematomas can mimic malignancy especially in cases of large and growing lesions occurring without a history of trauma. They appear as soft-tissue masses containing blood and a fibrous wall can be present.

On US examination acute/subacute hematomas appear illdefined, inhomogeneously hyperechoic while chronic collections tend to be well-defined and hypoechoic. On CT scans acute/subacute lesions are heterogeneous hyperdense masses while chronic lesions are usually hypodense with small calcifications.
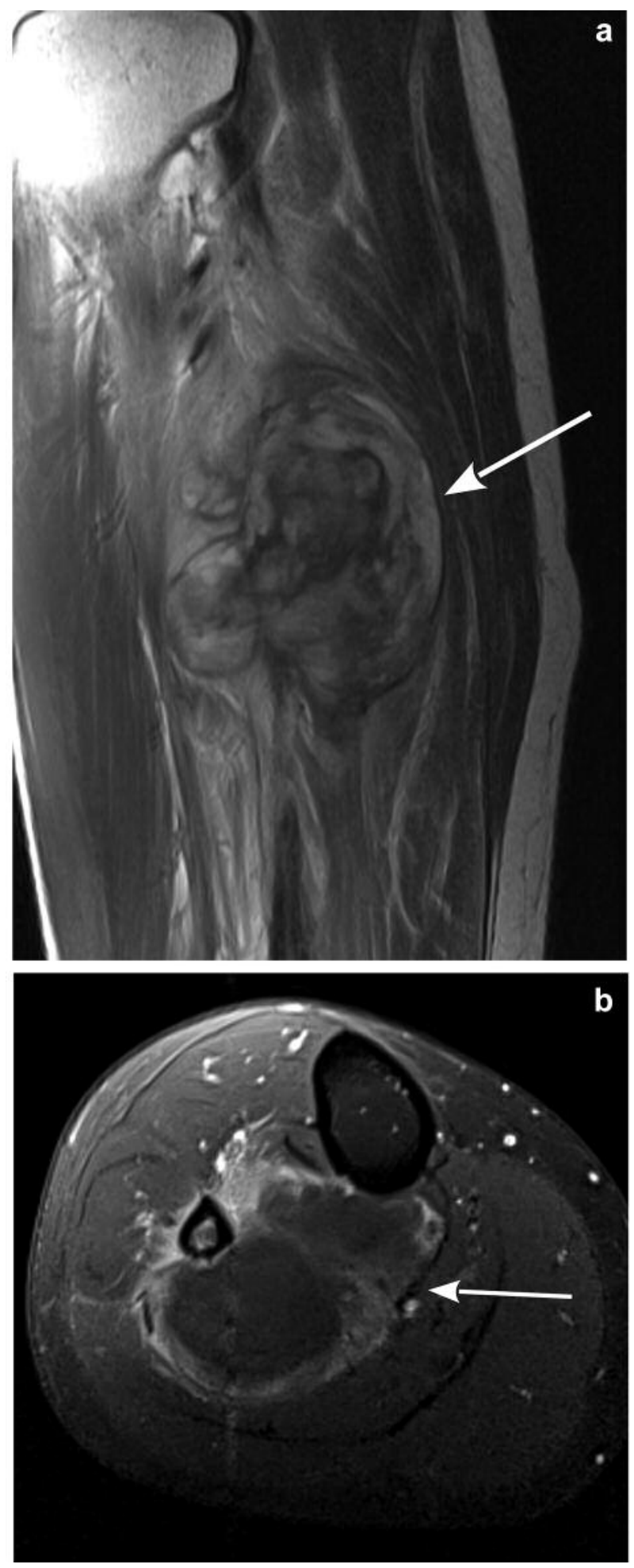

Figure 10. Hematoma: a) Sagittal Proton Density weighted MRI scan showing an inhomogeneous hyper-intense mass (white arrow) of the posterior compartment of the right leg; b) The same lesion (white arrow) in a T1 weighted axial MRI scan with fat saturation presenting peripheral contrast enhancement. Imaging follow-up demonstrated the typical MRI progression of hematoma. 

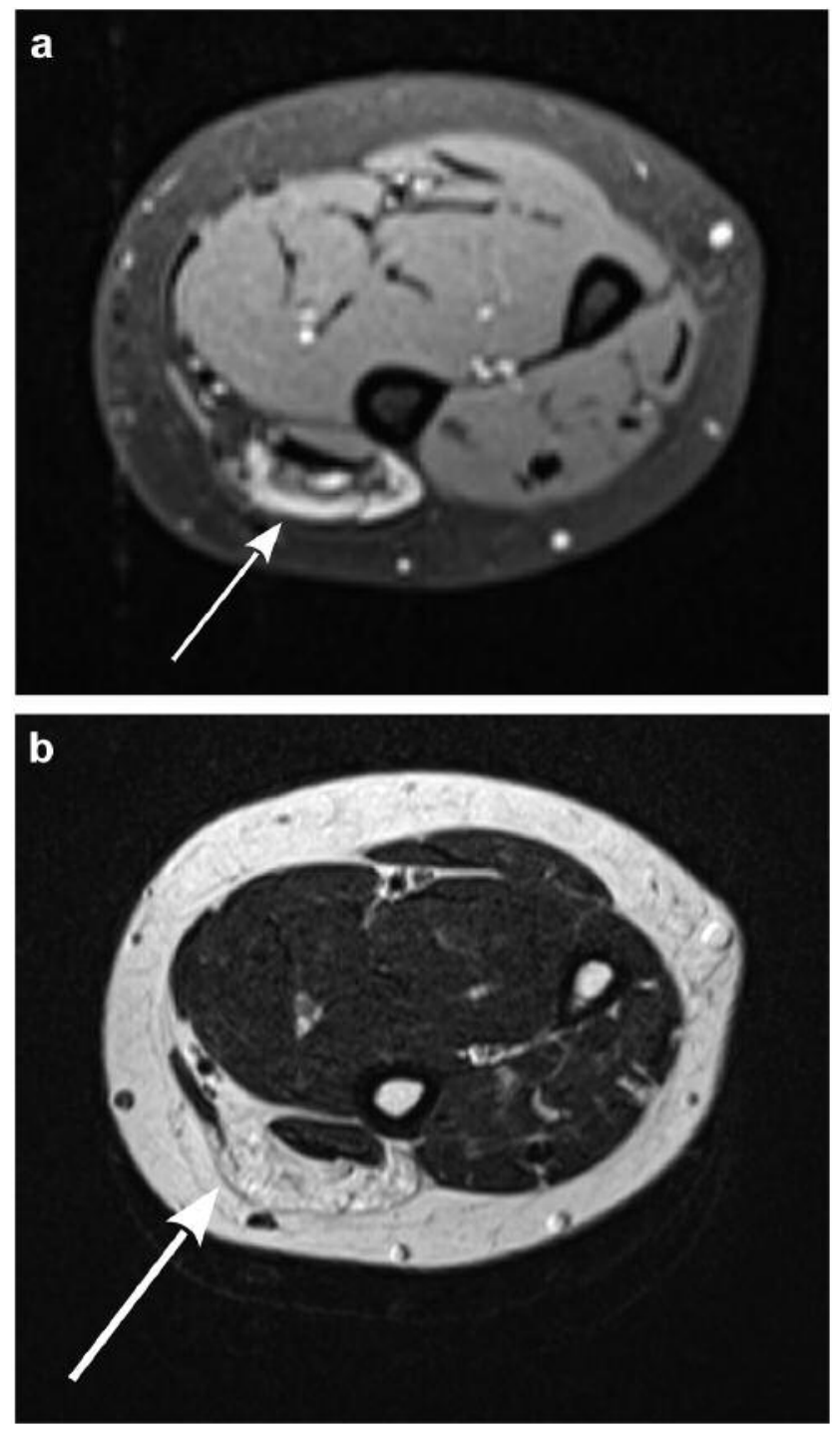

Figure 11. Arteriovenous malformation: a) Axial T2 weighted MRI scan showing a multi-lobulated lesion, between extensor carpi radialis muscles and brachioradialis muscle, that appears hyper-intense with flow-void inside high-flow vessels (white arrow); b) Axial T1 weighted MRI scan with fat signal suppression showing the same lesion with strong contrast enhancement of the abovementioned vessels (white arrow). Diagnosis of arteriovenous malformation was confirmed with both imaging and histopathology features.

As described above, hematomas are often inhomogeneous and MRI findings reflect that feature mainly depending on the hemoglobin breakdown phase. Acute lesions (0-2 days) usually present as heterogeneously hypointense masses in all sequences. Subacute hematomas, are hyperintese on T1 weighted images while on T2 weighted images they appear hypointense in the early subacute phase (2-7 days) and hyperintense in the late subacute phase (1-4 weeks);

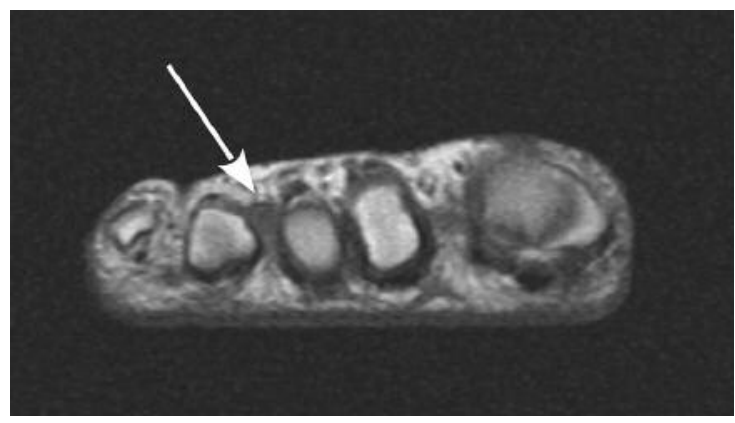

Figure 12. Morton's Neuroma: Axial T2 weighted MRI scan showing a fusiform lesion that appears hypo-intense compared to fat and hyperintense compared to muscles (white arrow), located in the third intermetatarsal space. Diagnosis of Morton's Neuroma was confirmed combining imaging and clinical features.

perilesional edema and peripheral rim enhancement may be present. Chronic hematomas ( $>4$ weeks) usually present as hypointese masses in all sequences and often showing fluid levels due to the separation of the cellular and fluid components (Figure 10) $(9,10)$.

Arteriovenous malformation ( $A V M)$. AVMs are non-neoplastic congenital vascular malformations considered as errors in vascular development. They consist of abnormal vessels with normal endothelium, composed of feeding arteries and a nidus of draining veins, without any capillary interposed.

Classified among high-flow malformations, they may arise in whole body and they usually grow gradually without regression, becoming significant during childhood or adulthood. Although they are all present at birth, they may not be detected until later in life due to slow growth. Trauma and hormonal influence at puberty and/or pregnancy can cause a more rapid growth of these lesions, mimicking a malignant lesion. On US examination they appear as anechoic tubular structures with variable Doppler-signal. CT scan show ill-defined masses with tangled arterial and venous structures; after intravenous iodine contrast injection an early contrast enhancement can be seen both in feeding arteries and drainage veins due to shunts. This is also evident on MR imaging, which also demonstrates the nidus as a tangle of prominent vessels with signal voids on T2 weighted images in vessels lumen because of the high-flow (Figure 11) (5). Perilesional edema with associated increased vascularity is not uncommon and that should not be mistaken for a solid neoplastic mass.

Morton's neuroma. Not real neuromas, Morton's Neuromas are non-neoplastic perineural fibrosing alterations of plantar digital nerves nearby metatarsal heads in the intermetatarsal space (usually 3rd) with high collagenous content. A strong predilection for women is seen $(\mathrm{F}: \mathrm{M}=18: 1)$. 
Morton's Neuromas are allegedly related to friction between nerve and transverse intermetatarsal ligament. US shows intermetatarsal fusiform and well-demarcated masses, generally hypo-echoic (although echotexture may vary), best appreciated after performing Mulder's test.

On MRI Morton's Neuromas appear hypo-isointense to muscles in T1 weighted images and hypo-intense to fat and hyper-intense to muscles in $\mathrm{T} 2$ weighted images; vivid enhancement is usually demonstrated after contrast administration. Corresponding intermetatarsal bursitis is often associated (Figure 12) (11).

\section{Conclusion}

We reviewed the most typical imaging features of soft tissue lesions mimicking malignancies that can be often encountered in daily radiologic practice. The interpretation of imaging findings however strongly requires a correlation with the clinical history and biopsy is still necessary in many cases for a final diagnosis.

\section{Conflicts of Interest}

The Authors have indicated they have no potential conflicts of interest to disclose.

\section{References}

1 Bridge JA, Hogendoorn P, Bridge JA and Fletcher CD: WHO classification of tumours of soft tissue and bone. International Agency for Research on Cancer, 2013.

2 McKenzie G, Raby N and Ritchie D: Non-neoplastic soft-tissue masses. Br J Radiol 82(981): 775-785, 2009.

3 Balik MS, Erkut A, Guvercin Y and Bedir R: Synovial Sarcoma Mimicking Myositis Ossificans. Knee Surg Relat Res 28(3): 249-252, 2016
4 Murphey MD, Vidal JA, Fanburg-Smith JC and Gajewski DA: Imaging of synovial chondromatosis with radiologic-pathologic correlation. Radiographics 27(5): 1465-1488, 2007.

5 Flors L, Leiva-Salinas C, Maged IM, Norton PT, Matsumoto AH, Angle JF, Hugo Bonatti M, Park AW, Ahmad EA, Bozlar U, Housseini AM, Huerta TE and Hagspiel KD: MR imaging of soft-tissue vascular malformations: diagnosis, classification, and therapy follow-up. Radiographics 31(5): 1321-1340, 2011.

6 Dinauer PA, Brixey CJ, Moncur JT, Fanburg-Smith JC and Murphey MD: Pathologic and MR imaging features of benign fibrous soft-tissue tumors in adults. Radiographics 27(1): 173187, 2007.

7 Stramare R, Beltrame V, Gazzola M, Gerardi M, Scattolin G, Coran A, Faccinetto A, Rastrelli M and Rossi CR: Imaging of soft-tissue tumors. J Magn Reson Imaging 37(4): 791-804, 2013.

8 Petscavage-Thomas JM, Walker EA, Logie CI, Clarke LE, Duryea DM and Murphey MD: Soft-tissue myxomatous lesions: review of salient imaging features with pathologic comparison. Radiographics 34(4): 964-980, 2014.

9 Crundwell N, O'Donnell P and Saifuddin A: Non-neoplastic conditions presenting as soft-tissue tumours. Clin Radiol 62(1): 18-27, 2007.

10 Kwok HC, Pinto $\mathrm{CH}$ and Doyle AJ: The pitfalls of ultrasonography in the evaluation of soft tissue masses. J Med Imaging Radiat Oncol 56(5): 519-524, 2012.

11 Murphey MD, Smith WS, Smith SE, Kransdorf MJ and Temple HT: From the archives of the AFIP. Imaging of musculoskeletal neurogenic tumors: radiologic-pathologic correlation. Radiographics 19(5): 1253-1280, 1999.
Received December 28, 2017

Revised January 19, 2018

Accepted January 23, 2018 